\title{
Compromise in vectorcardiography Displacement of electrodes as a means of adapting one lead system to another
}

\author{
II. C. Burger, D.SC. \\ A. G. W. van Brummelen, M.S. \\ G. van Herpen, M.D. \\ Utrecht, Netherlands
}

$\mathrm{T}$ he variety of systems of vectorcardiography, and the discrepancies existing between various systems, even among those with a well-founded physical basis, have impeded full acceptance of vectorcardingraphy for clinical use. Standardization is urgently needed, but cannot be realized yet at the present time.

One thing is certain: all systems without rational physical foundation must be abandoned. If two systems differ, although both have a sound physical foundation, a step toward standardization may be taken if a compromise between the two systems can be found. ${ }^{1}$

A lead system is characterized, first, by the choice of the electrode positions, and, second, by the manner in which the voltages are combined with the use of coefficients (or lead vectors). Thus, there are two methods of making a compromise between two systems, viz., by proper change of the coefficients or by displacement of the electrodes. Since instruments which allow a free choice of the coefficients are not generally used, we chose to investigate the second method.

The systems investigated were Schmitt's SVEC-II ${ }^{2}$ and our $\mathrm{B}_{1} \mathrm{~W}_{4}{ }^{\prime 3,4}$ which we will indicate here by the letters $\mathrm{S}$ and $\mathrm{B}$, respectively. Inasmuch as we have only recently become aware that our system has never been described explicitly, we wish to present its technical particulars here. The following electrode positions are used: right arm (R), left arm (L), left leg $(\mathrm{F})$, chest, a mid-sternal electrode placed at the level of the axillae (B) and back, the dorsal electrode of Wilson's tetrahedron, $2 \mathrm{~cm}$. to the left of the seventh thoracic vertebra (W).

Since the number of independent combinations of electrodes amounts to one less than the number of electrodes, 4 leads are obtained from these 5 positions. The leads are so chosen that only one electrode (R) is combined alternatively with each of the others, yielding the leads LR, FR, BR, WR. The lateral, longitudinal, and sagittal components* of the vector are obtained by combining the leads with one another and assigning them with coefficients determined by model experiments.

$$
\begin{aligned}
& \mathrm{X}=56 \mathrm{LR}+16 \mathrm{FR}+4 \mathrm{BR}-9 \mathrm{WR} \\
& \mathrm{Y}=-9 \mathrm{LR}+26 \mathrm{FR}-3 \mathrm{BR}+7 \mathrm{WR} \\
& Z=9 \mathrm{LR}-27 \mathrm{FR}-20 \mathrm{BR}+40 \mathrm{WR} \\
& \text { (LR = potential of } \mathrm{L}-\text { potential of } \mathrm{R}, \\
& \text { etc.) }
\end{aligned}
$$

It is evident that any alteration in the positioning of even one electrode in this system would result in a change of the value of all 3 components of the vector.

\footnotetext{
From the Department of Medical Physics, Physical Laboratory of the University of Utrecht, Utrecht, Netherlands. Received for publication Feb. 20, 1961.

*The American notation has been followed, in which the axes are chosen as follows: $\mathrm{X}=$ right,- left $+\mathrm{Y}=\mathrm{cranial}$ - caudal $+; Z=$ ventral - , dorsal + .
} 
This, in turn, would require new values for the coefficients. In the S-system, each component of the vector is determined by one single lead. Consequently, we have chosen to adapt the S-system to the Bsystem and not the reverse. This does not mean, however, that we attribute the difference of these two systems to an error in Sonly.

\section{Method and material}

In the Schmitt system there is no mention of a scale, so that we have chosen the sensitivity in such a manner that the sizes of the loops in the $\mathrm{S}$ and $\mathrm{B}$ systems were, on the average, approximately equal. The frontal and horizontal projections in each system were photographed simultaneously.

The agreement between the frontal projections of the two systems is satisfactory; the main discrepancy is in the horizontal projection, and is the consequence of the uncertainty in the determination of the sagittal component of the heart vector, caused by the relatively small dimensions of the human thorax in the sagittal direction.

It appeared that in the S-system a footward loop generally is directed more posteriorly than in the B-system. This was expressed previously ${ }^{3}$ in an analytical form by the relation:

$$
Z_{\mathrm{S}}=-0.2 \mathrm{X}_{\mathrm{B}}+0.6 \mathrm{Y}_{\mathrm{B}}+0.9 \mathrm{Z}_{\mathrm{B}}
$$

in which $Z_{\mathrm{s}}$ is the sagittal component of the vector in the $S$-system, and $X_{B}, Y_{B}$, and $Z_{B}$ are the components in the B-system. Because of the contribution of the Y-term, which is positive for points of the vector loop below the zero point, $Z_{\mathrm{s}}$ will become proportionately greater than $Z_{\mathrm{B}}$ at points which are increasingly distant from the zero point, moving in a caudal direction.

The sagittal component in the S-system is exclusively determined by, and proportional to, the voltage between its dorsal and precordial electrodes. Consequently, the desired change in this component might be attempted by shifting these two electrodes. The anatomic axis connecting these electrodes changes, therefore, in position: we choose to call this axis the "pick-off" axis. If the dorsal electrode is displaced cranially and the precordial electrode caudally, an inclination of the sagittal pick-off axis is effected. This will bring about a decrease in the magnitude of the projection on the axis of a caudo-posteriorly directed vector, and an increase in the case of a caudo-anteriorly directed vector. The net result would be a rotation of the vector loop in the ventral direction, over a certain angle (see Fig. 1). This reasoning would only be fully justified if image space and anatomic space were considered to be identical, which is certainly not true. Preliminary investigations, however, have supported the above-mentioned displacement of electrodes; the distance was empirically established at $6 \mathrm{~cm}$. for each electrode.

Our material comprised 64 normal individuals and 86 cardiac patients. Comparisons were made by three observers independently, by grading the agreement between the horizontal projections of the loops according to a scale ranging from 0 to $10 . \Lambda$ score of 10 indicated as perfect an agreement as could be expected to exist within one system between different heart beats of the same subject...$^{3-5}$ An example of the improvement in agreement between systems $B$ and $S$ is shown in Fig. 2.

\section{Results and conclusions}

From the group material the average of the scores obtained from the comparison

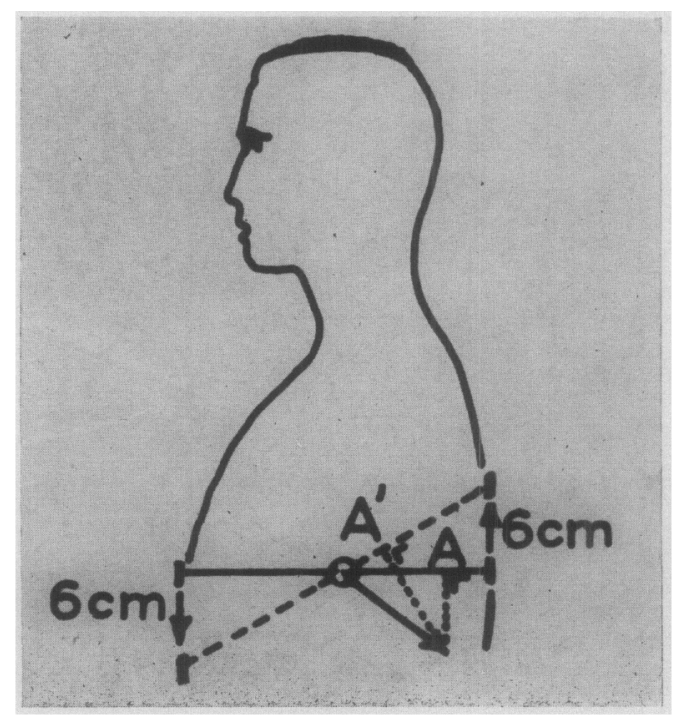

Fig. 1. $O A$ represents the projection of the heart vector on the pick-off axis before, and $O A^{\prime}$ after, displacement of the electrodes. It can readily be seen that $\mathrm{OA}^{\prime}<\mathrm{OA}$ for posteriorly directed vectors. 


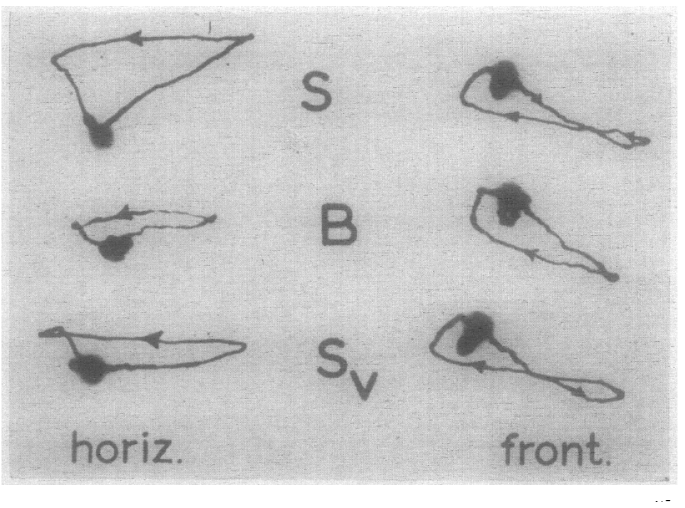

Fig. 2. $S_{r}$, the loop obtained after displacement of the electrodes, shows a better resemblance to $B$ in the horizontal projection than does $S$.

between $B$ and the original $S$ and its variant $S_{V}$, respectively, revealed a value of $6.0 \pm$ 0.12 for $\mathrm{B}-\mathrm{S}$ and $6.5 \pm 0.12$ for $\mathrm{B}-\mathrm{S}_{\mathrm{V}}$. The average of the individual differences between $\mathrm{B}-\mathrm{S}$ and $\mathrm{B}-\mathrm{S}_{\mathrm{V}}$ was $0.5 \pm 0.07$. This difference is statistically significant, but the improvement is hardly large enough to render the method worth while for practical use in its present form. The failure of this result to fulfill our expectations may be attributed to: (1) the application of a rotation instead of a shear, which would be required considering the above-mentioned formula for $Z_{S} ;(2)$ the assumption that the discrepancies between $S$ and $B$ are due to a difference in the sagittal components only and can be remedied by changing these components only; (3) the assumption that image space and anatomic space are identical.

Therefore, we have abandoned this method and have turned our attention to the procedure of changing the coefficients.

\section{Summary}

An attempt was made to improve the agreement between the Schmitt SVEC-II and the Burger $B_{1} W_{4}^{\prime \prime}$ systems by shifting the ventral and dorsal electrodes of the SVEC-II system caudally and cranially, respectively. Although a statistically significant improvement in agreement was obtained, it hardly warrants practical use of the procedure.

\section{REFERENCES}

1. Burger, $H, C$., van Brummelen, A. G. $W$., and van Herpen, G.: Heart-vector and leads, Am. Heart J. 6l:317, 1961.

2. Schmitt, O. H., and Simonson, E.: The present status of vectorcardiography, A.M.A. Arch. Int. Med. 96:574, 1955.

3. Burger, H. C., van Milaan, J. B., and Klip, W.: Comparison of three different systems of vectorcardiography, AM. HEART J. 57:723, 1959.

4. Burger, H. C., van Milaan, J. B., and Klip, W. Comparison of two systems of vectorcardiography with an electrode to the frontal and dorsal sides of the trunk, respectively, Am. Heart J. 51:26, 1956.

5. Burger, H. C., van Milaan, J. B., and den Boer, W.: Comparison of different systems of vectorcardiography, Brit. Heart J. 14:401, 1952. 